\title{
Characterization and Detailed Analysis of Regression Behavior for HTPB Solid Fuels Containing High Aluminum Loadings
}

\author{
Timothy P. Kibbey ${ }^{1}$ \\ Jacobs ESSSA Group, Huntsville, AL 35812 \\ Andrew C. Cortopassi ${ }^{2}$ and J. Eric Boyer ${ }^{2}$ \\ Pennsylvania State University, University Park, PA 16802
}

\section{Extended Abstract}

\section{A. Introduction}

NASA Marshall Space Flight Center's Materials and Processes Department, with support from the Propulsion Systems Department, has renewed the development and maintenance of a hybrid test bed for exposing ablative thermal protection materials to an environment similar to that seen in solid rocket motors (SRM). The Solid Fuel Torch (SFT), operated during the Space Shuttle program, utilized gaseous oxygen for oxidizer and an aluminized hydroxylterminated polybutadiene (HTPB) fuel grain to expose a converging section of phenolic material to a $400 \mathrm{psi}$, 2-phase flow combustion environment. The configuration allows for up to a 2 foot long, 5 inch diameter fuel grain cartridge. Wanting to now test rubber insulation materials with a turn-back feature to mimic the geometry of an aft dome being impinged by alumina particles, the throat area has now been increased by several times to afford flow similarity. Combined with the desire to maintain a higher operating pressure, the oxidizer flow rate is being increased by a factor of 10. Out of these changes has arisen the need to characterize the fuel/oxidizer combination in a higher mass flux condition than has been previously tested at MSFC, and at which the literature has little to no reporting as well.

For (especially) metalized fuels, hybrid references have pointed out possible dependence of fuel regression rate on a number of variables:

- $\quad$ mass flux, $\mathrm{G}$

- $\quad$ oxidizer only $\left(\mathrm{G}_{0}\right)$, or

- total mass flux $\left(\mathrm{G}_{\mathrm{tot}}\right)$

- Length, L

- Pressure, $\mathrm{P}$

- Diameter, D

In Sutton ${ }^{1}$, Boardman suggests the form

$$
\dot{r}=f\left(G_{0}, L,\left(1-e^{-\frac{P}{P_{r e f}}}\right),\left(1-e^{-\frac{D}{D_{r e f}}}\right)\right)
$$

Clearly, these factors themselves could vary depending on the aluminum loading of the fuel. For this particular endeavor, aluminum loading level is important to investigate, as previous SFT operation used solely $18 \%$-aluminumloaded fuel grains, while $40 \%-50 \%$ is more appropriate for matching an SRM's combustion products content. The diameter will be important for two reasons: the subscale testing selected for this initial characterization at Penn State University's High Pressure Combustion Lab (HPCL) operates at diameters of 0.25-1.25 inches, while the SFT will operate at higher diameters, and the SFT itself will scan the diameter range from 1.5-4 inches.

\footnotetext{
${ }^{1}$ Ballistics - Solid Launch Systems \& Analysis, AIAA Member.

${ }^{2}$ Research Associate, High Pressure Combustion Lab, Department of Mechanical and Nuclear Engineering, AIAA Member.
} 
Karabeyoglu ${ }^{2}$ suggests a different function for average regression rate including mass flux, length and diameter for a non-metalized grain. It is thought that the pressure effect is important for metalized fuel grains due to radiation, and Lund $^{3}$ reported the form

$$
\dot{r}=a G^{n} P^{m}
$$

with $\mathrm{m}$ as high as 0.36 for $61 \%$ aluminum loading. Estey ${ }^{4}$, et. al., compute 9 different functional forms for some data, but none (except the Sutton/Boardman form) includes both length and diameter together.

The exact relation between all the variables is still quite undetermined, so the more data available on their potential interactions, the better. For instance, is the average regression based on L \& D independently, or D and L/D?

Typically, the Reynolds number has been found in some basic calculations, and either length or diameter supplied where the "distance" dimension belongs, thinking of regression as either a boundary layer or pipe flow issue. However, it is very likely an interaction of both, with the relationship between the parameters determining the exact interplay.

Consider the oxidizer flow entering the fuel grain: it initially attaches a growing boundary layer, which can be characterized with an axial Reynolds number, analogous to flat plate friction and heat transfer. However, this really just acts as a pipe entrance length: at some point downstream, the momentum boundary layers converge at the centerline, at a distance determined by the diameter. After this point, the flow may be better characterized with the pipe Reynolds number including diameter, being fully developed, albeit with axial velocity increase due to fuel insertion into the flow. In addition, there is a transition from laminar to turbulent flow somewhere in there, and finally, for long enough grains, if the Ox to fuel ratio becomes sufficiently low, the combustion zone temperature may drop, further changing the characteristics of the aft-most flow.

The data of Ref. 5 bear this out: See Figure 1to the right, where for several test conditions the first and last segments regressed the most, with the middle two segments at lower regression rates.

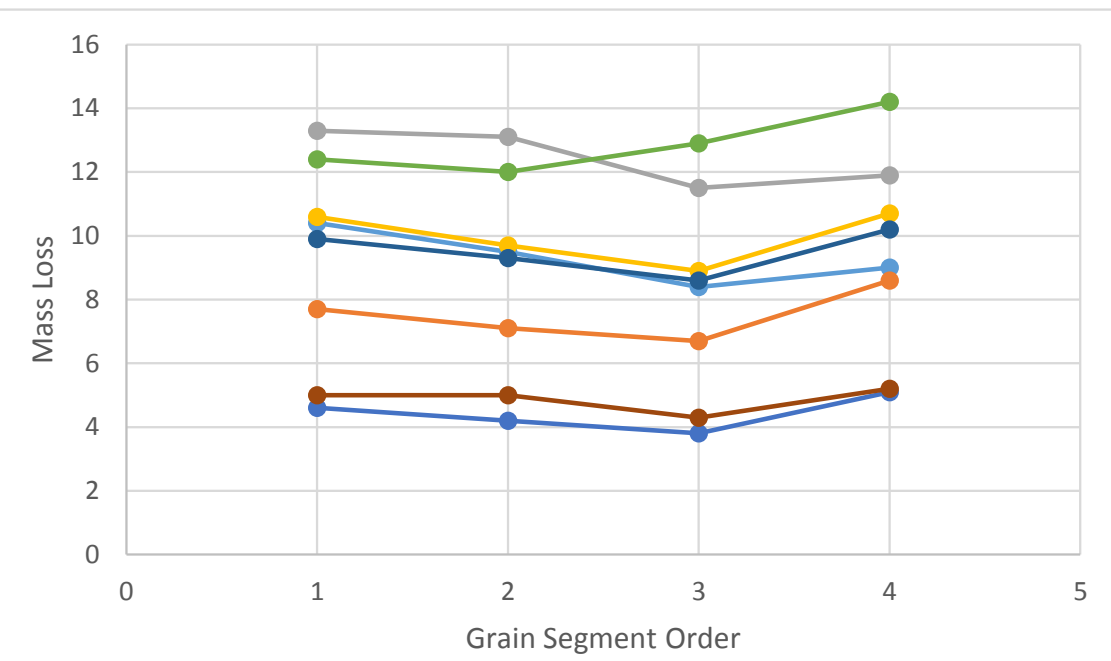

Figure 1. Test data from the NASA Large Subscale Solid Rocket Combustion Simulator Program (from Ref. 5)

\section{B. Experimental Method}

The High Pressure Combustion Laboratory (HPCL) at the Pennsylvania State University's University Park campus has extensive hybrid rocket test facilities available. One of the hybrid rocket motors with specialized capabilities, along with its associated oxidizer (gaseous $\mathrm{O}_{2}$ or GOX) feed systems, and control and data acquisition systems was utilized in this screening program: the long-grained center-perforated (LGCP) hybrid rocket motor (see Figure 2). The highly robust LGCP is a suitable motor for initial screening and evaluation of solid fuel grains with novel port designs or formulations in combination with the selected oxidizer. The relatively small size (grain outer diameter $\sim 1.5 "$ ) allows characterization tests to be performed with minimal material consumption. The LGCP rocket motor is instrumented for pressure and thrust measurements, a straight injector was used and the nozzle throat sizes was varied to tailor the chamber pressure with varying oxidizer flow rates and fuel mass production rates. Gaseous oxygen (GOX) was used as the oxidizer for the hybrid rocket motor tests. All flow-control valves and igniter signals are remotely controlled by a computer program. For use in the analyses discussed here, high-resolution time-series data $(1 \mathrm{kHz})$ for chamber pressure and oxidizer mass flow rate were measured. 

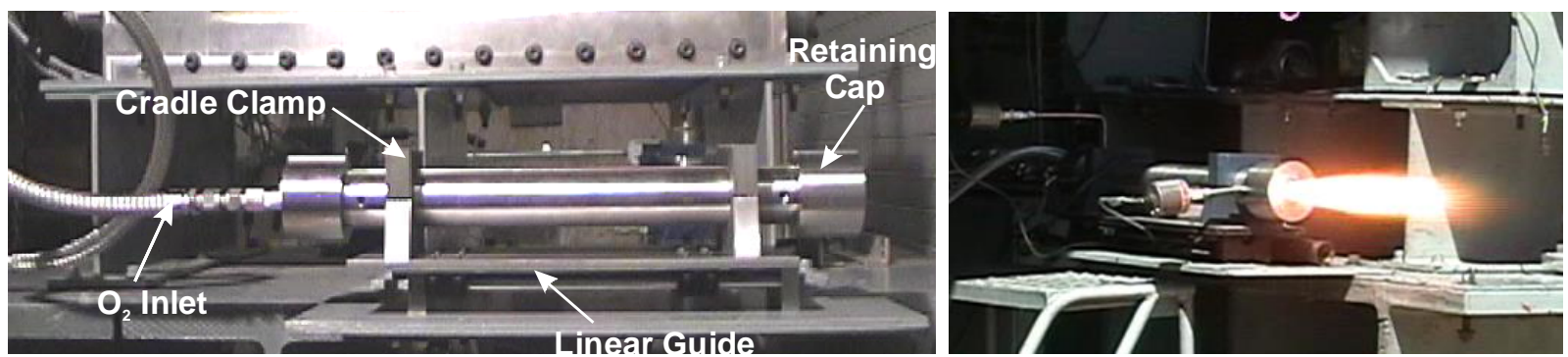

Figure 2. The LGCP hybrid rocket motor at Penn State's High Pressure Combustion Lab.

The methods used to compute bulk average parameters by integrating the pressure-time data, and the examination of the axial position data will be included in the final paper.

\section{DOE Plan}

After the initial exploratory runs, the program had a budget of 16 remaining characterization tests at HPCL. Testing different-length grains was discussed, but from the initial exploratory testing, shorter grains led to too high an oxidizer-to-fuel ratio $(\mathrm{O} / \mathrm{F})$, which in this configuration leads to disadvantageous strong throat erosion. Longer grains would depart more from the SFT length-to-diameter ratio (L/D) and be more difficult to cast. So it was decided to ascertain axial position effects from dissection of the grains rather than by using overall grain length as a parameter in the design of experiments. That left a two-level, full factorial design available over Aluminum loading level, mass flow rate, pressure, and diameter. A fully successful test matrix would allow estimation of all main effects, 2-way, and 3-way interactions, or all main effects and 2-way interactions with 5 Error Degrees of Freedom (DoF). If any have to be thrown out due to data anomalies, etc., statistical assessments can still lead to good quality assessments, just with some slight correlation with some of the interactions. However, probably not all two way interactions will be important, thus further increasing the error DoF.

The full factorial layout allows every combination of parameters to be tested. Additionally, care was taken to ensure that if early tests encountered problems, the remaining data could be of the highest value. By using statistical software to set up a 12-run "Irregular Fraction" DOE, it was found that the runs denoted "DOE line $2,3,5,8$ " were not required in the 12-run analysis. Therefore, if those were done first, and had erroneous data, the 12-run analysis could still be substituted for the desired 16-run analysis.

\section{DOE Carried Out}

The Aluminum loading level and oxidizer mass flow rate can be set exactly, along with the initial grain diameter. However, because there is no real-time regression monitoring, much weight will

Table 1. DOE matrix for regression testing

\begin{tabular}{||c|cccc|r|}
\hline \multirow{2}{*}{ Test Order: } & Alum & Pressure & mdot_ox & Diam & DOE line \#: \\
\cline { 2 - 5 } $1-4$ & $18 \%$ & 400 & high & 0.375 & 3 \\
$1-4$ & $18 \%$ & 600 & high & 0.5 & 8 \\
$1-4$ & $18 \%$ & 600 & low & 0.375 & 5 \\
$1-4$ & $18 \%$ & 400 & low & 0.5 & 2 \\
& $18 \%$ & 600 & high & 0.375 & 7 \\
& $18 \%$ & 600 & low & 0.5 & 6 \\
& $40 \%$ & 600 & low & 0.5 & 14 \\
& $40 \%$ & 400 & low & 0.5 & 10 \\
& $40 \%$ & 600 & low & 0.375 & 13 \\
& $40 \%$ & 600 & high & 0.375 & 15 \\
& $40 \%$ & 400 & high & 0.5 & 12 \\
& $40 \%$ & 400 & low & 0.375 & 9 \\
& $18 \%$ & 400 & low & 0.375 & 1 \\
& $40 \%$ & 400 & high & 0.375 & 11 \\
& $18 \%$ & 400 & high & 0.5 & 4 \\
& $40 \%$ & 600 & high & 0.5 & 16 \\
\hline
\end{tabular}

have to be given to time-averaged values during data analysis and modeling. Because of this, the average pressure and average diameter will be important; guaranteeing that these come out similar for the targets above will require good prediction. The actual controls for these values are the throat area and burn time. The first four tests were an 
invaluable proving ground; for the remaining 12, the targeting of pressure and average diameter was much tighter, as shown in Figure 3.

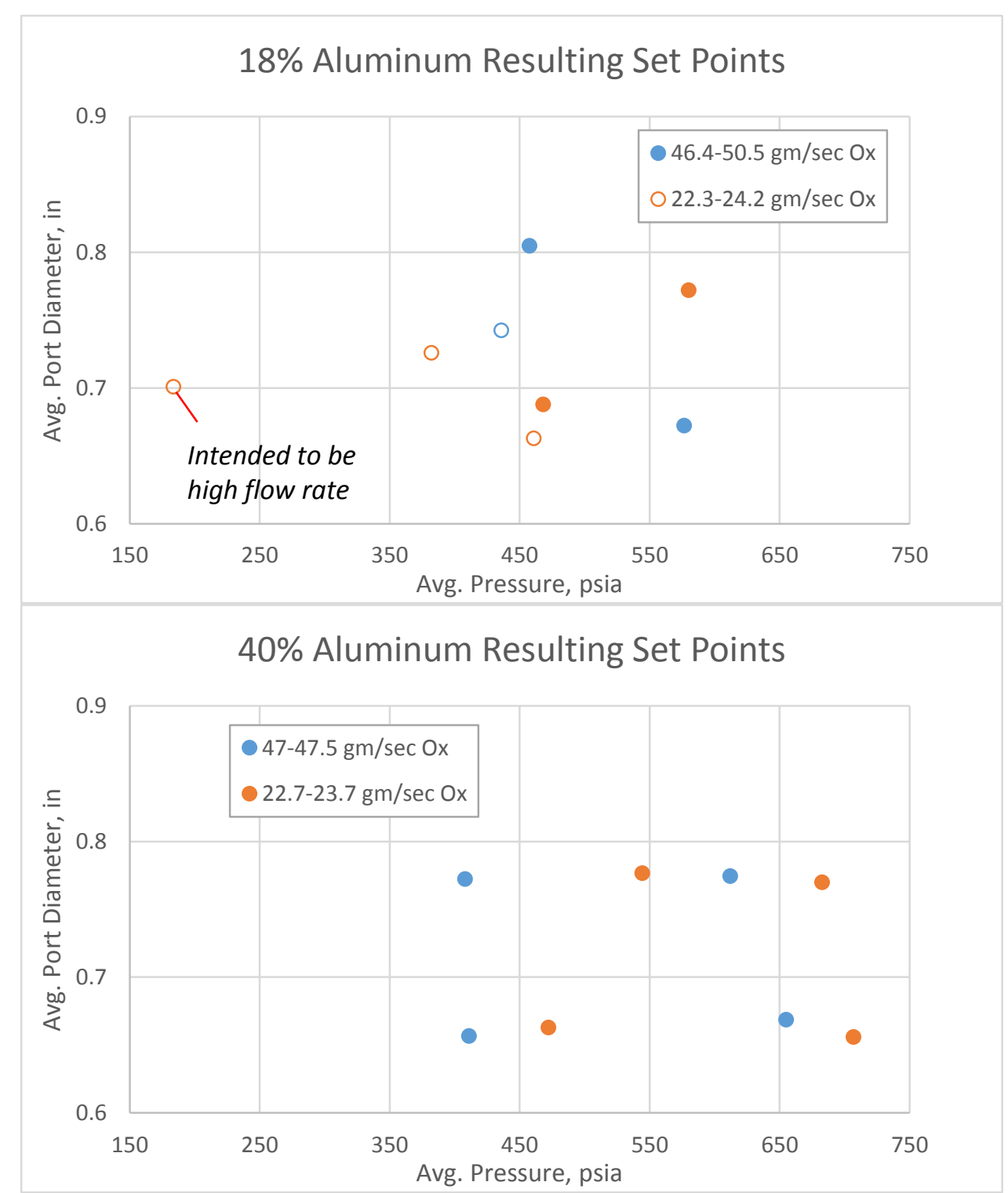

Figure 3. Actual operating conditions for tests conducted following the DoE plan.

Note that, for all 12 points as closed circles, "high" and "low" are delineated, even though pressure is not necessarily grouped tightly. This will be sufficient to provide orthogonality between the factors when modeling. Similarly with oxidizer flow rate: even though the average mass flux is a derived parameter not at exactly 2 levels, the independence of the high and low flow rates in combination with the other variables guarantees orthogonality and assigns the output variable's dependence appropriately.

\section{E. Data and Analysis:}

\section{Regression based on mass burned}

The initial look at the data is by computing the average regression rate that would result in the measured mass loss. As suggested by Karabeyoglu ${ }^{2}$, this is applied at the "average mass flux" defined as oxidizer mass flow divided by area at the average diameter. This allows examination of combustion efficiency at modeling of regression rate (shown in Figure 4). Most $\mathrm{c} *$ efficiencies are greater than $92 \%$ for the $18 \%$-aluminum fuel, and greater than $89 \%$ for the $40 \%$ - 
aluminum fuel. (The open circle point is Test 14 , which had a long blowdown due to a valve timing issue.) This is acceptable and consistent performance for a test article of this size, and having to deal with significant nozzle erosion on some tests.

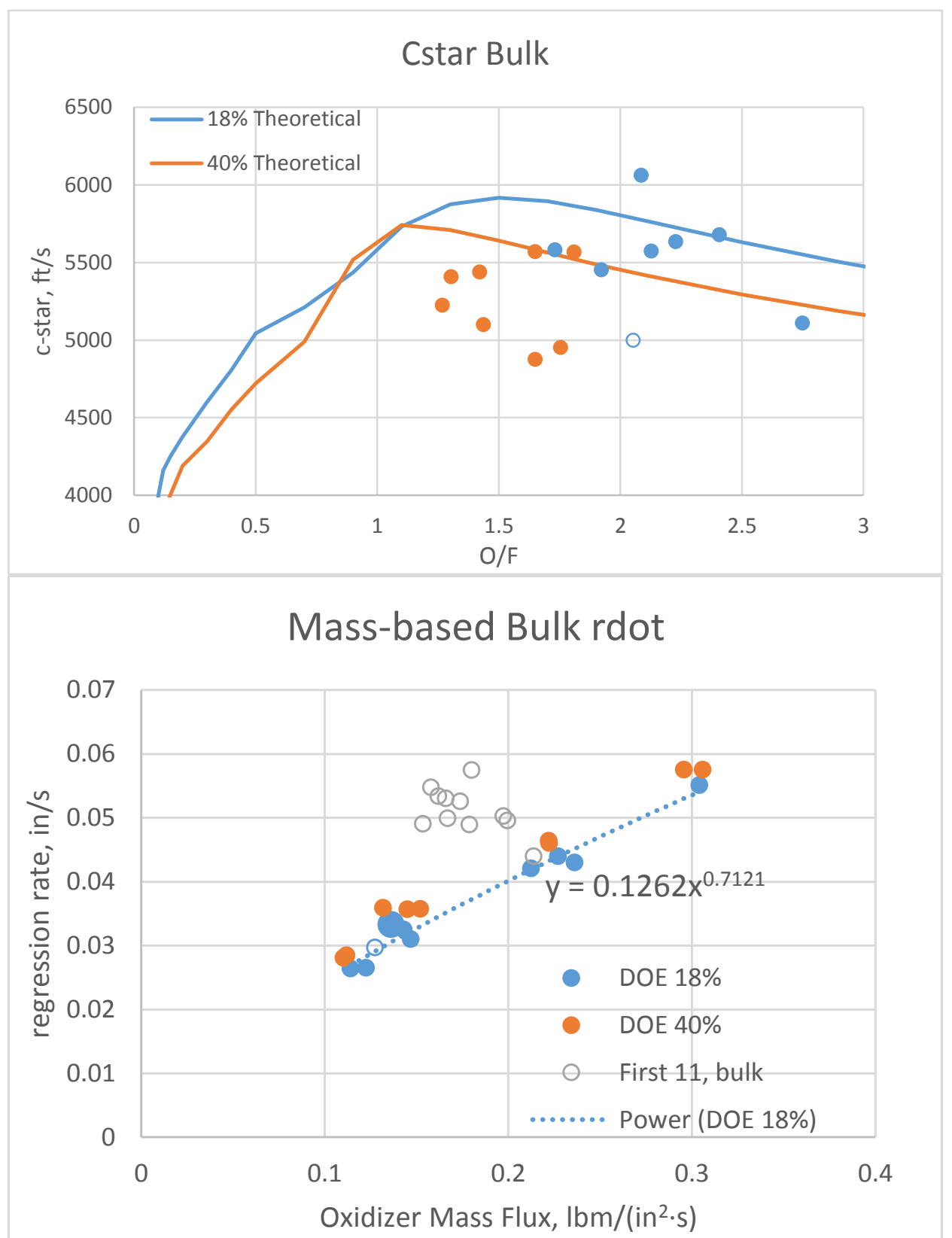

Figure 4. Calculated $\mathrm{c}^{*}$ combustion values and regression rates for experiments.

By inspection of the plotted regression rate data (where the mass flux is based on oxidizer flow rate), a small but significant increase in regression rate was experienced by jumping to the $40 \%$ aluminum loading. Also, the mass flux range tested was greatly increased; a 3:1 ratio of max:min mass flux should give high confidence in calculations of its exponent. Finally, note the open circles named "First 11, bulk." These were the exploratory tests that had issues with internal strain cracks. They have a significantly higher effective bulk regression rate because the opened surface area and turbulent flow allowed for much more mass extraction and burning. However, this should not be thought of as a steady regression phenomenon: the image below shows a post-burn example where the additional mass came from local zones influenced by the initial cracks. 


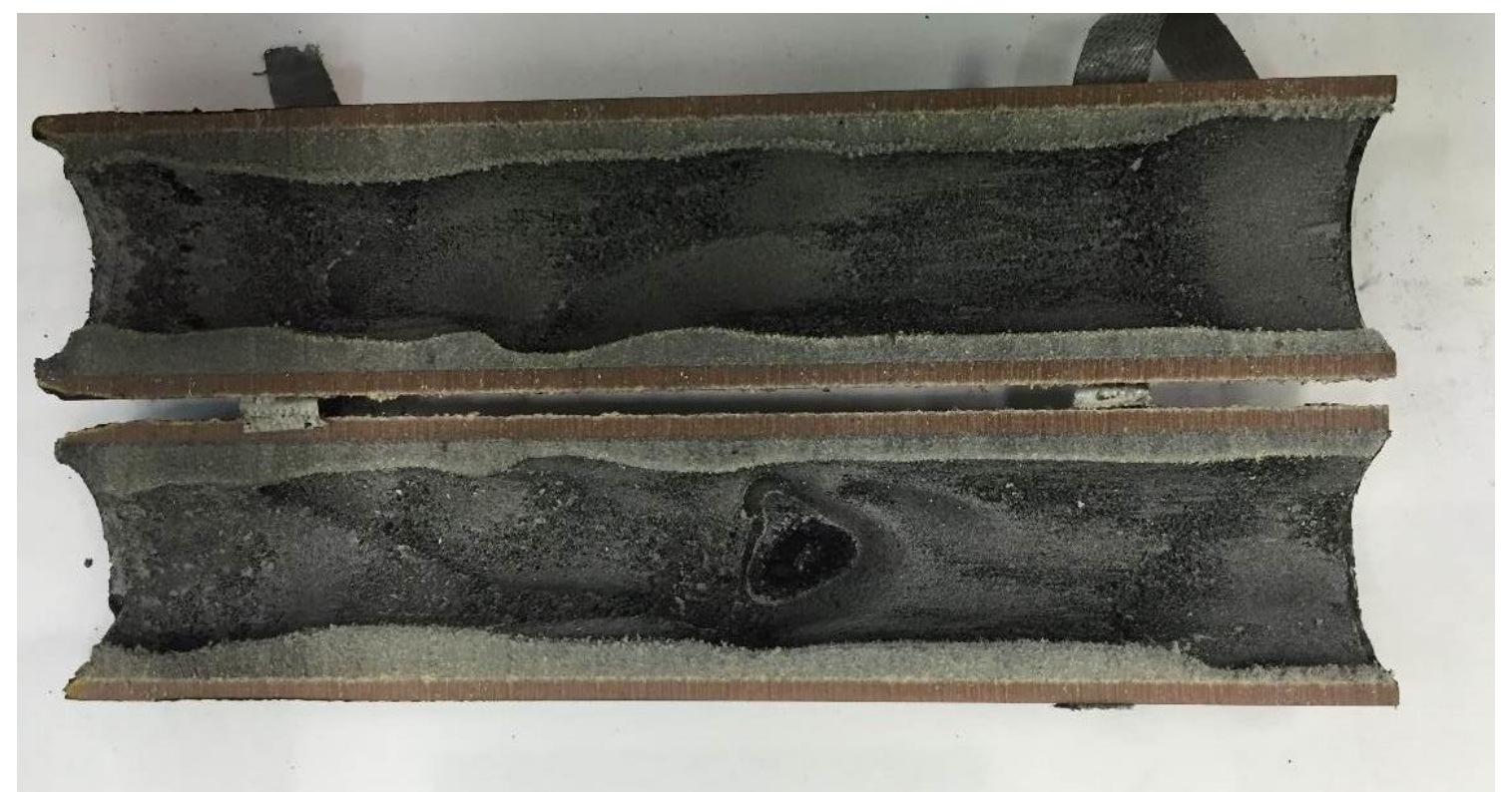

Figure 5. Post-test sectioned view of fuel grain that contained initial strain cracks/tears.

To begin modeling the data, first consider just a multiple-linear regression of the $40 \%$-Aluminum data. A hypothesized working correlation

$$
\dot{r}_{40 \%}=a_{40 \%} G^{n} P^{m} D^{l}
$$

can be converted to a linear form as:

$$
\log (\dot{r})=\mathrm{b}+\mathrm{m}_{\mathrm{P}} \log (\mathrm{P})+\mathrm{m}_{\mathrm{G}} \log (\mathrm{G})+\mathrm{m}_{\mathrm{D}} \log (\mathrm{D})
$$

The computed parameters are as follows in Table 2:

Table 2. Regression rate parameters for Equation 4 for $40 \%$ aluminum tests.

\begin{tabular}{|c|c|c|c|c|}
\hline & $\log \mathrm{P}$ & $\log \mathrm{G}$ & $\log \mathrm{D}$ & $\mathrm{b}$ \\
\hline Slope, $\mathrm{m}_{\mathrm{n} . . .0}$ & -0.01 & 0.69 & -0.13 & -2.02 \\
\hline Standard error of slope, $\mathrm{se}_{\mathrm{n} . .0}$ & 0.03 & 0.02 & 0.08 & 0.18 \\
\hline
\end{tabular}

To see the significance of factors, compare the absolute values of the slopes $m$ to their standard error se. As expected, the mass flux effect is very strong $(0.69>>0.02)$, and its value is near 0.7. Previous publications had shown values anywhere from 0.33 to 0.93 for HTPB and aluminized HTPB with gaseous oxygen, so this alone is an important bit of information. Secondly, the diameter effect appears significant, and constitutes an exponent of around $-1 / 8$. This is the correct sign qualitatively, in that larger diameter is expected to reduce the regression rate. Finally, the pressure effect is insignificant. Further examinations of the 18\%-Aluminum data also show insignificant pressure effect. This is a key finding, that any radiation effects due to aluminum do not lead to a pressure effect, and will simplify the further analysis that includes axial effects.

\section{Regression measured at head and aft}

The next data taken and analyzed was the measured regressed diameter at each end of the grain. At the head end (Table 3), this is typically the minimum diameter axially, to avoid the end effect of rounding off the entrance corner. Similar care was taken at the aft end (Table 4) to be consistent. This was then computed into average regression rate. The same equation as above can be used, where now the mass flux will be the total mass flux for the aft end (for forward end, the total mass flux simply is the oxidizer mass flux). 
Table 3. Regression rate parameters for head-end.

\begin{tabular}{|c|c|c|c|c|}
\hline & $\log \mathrm{P}$ & $\log \mathrm{G}$ & $\log \mathrm{D}$ & $\mathrm{b}$ \\
\hline Slope, $\mathrm{m}_{\mathrm{n} \ldots 0}$ & 0.08 & 0.64 & 0.25 & -2.79 \\
\hline Standard error of slope, $\mathrm{se}_{\mathrm{n} \ldots 0}$ & 0.09 & 0.06 & 0.22 & 0.57 \\
\hline
\end{tabular}

Table 4. Regression rate parameters for aft-end.

\begin{tabular}{|l|l|l|l|l|}
\hline & $\log \mathrm{P}$ & $\log \mathrm{G}$ & $\log \mathrm{D}$ & $\mathrm{b}$ \\
\hline Slope, $\mathrm{m}_{\mathrm{n} \ldots 0}$ & -0.02 & 0.79 & -0.19 & -2.05 \\
\hline Standard error of slope, $\mathrm{se}_{\mathrm{n} . .0}$ & 0.07 & 0.05 & 0.22 & 0.42 \\
\hline
\end{tabular}

Now notice not only the lower significance of the diameter term, but on the head-end, it switches signs! The aft still seems consistent with the bulk, qualitatively at least.

\section{Initial theory of axial position:}

To understand this difference between forward and aft end, it is possible that those modelers who have chosen the flat plate boundary-layer growth analogy are correct, to a point. Near the beginning of a tube, the boundary layer grows, but this only lasts as long as an entry length. So perhaps the axially-based regression rate formulations are valid for the initial length of fuel grain, but at some $\mathrm{x} / \mathrm{D}$ transition to a rate law that accounts for diameter change is required.

This theory will be examined more, and hopefully quantified, in the final paper, with inclusion of the axial data from dissecting the grains. As additional clarification, both these tests and the target operational size of the SFT have single-port axial injection of the gaseous oxidizer; the entrance and axial characteristics to be determined might be limited to only such a configuration.

\section{F. Conclusion}

The data taken significantly surpasses the previous available data on regression rate of aluminized HTPB fuel burning with gaseous oxygen. It encompasses higher mass fluxes, and appears to generate more consistent data. See Figure 6 below for evidence of that. The good test article and facility design and testing work of the Penn State HPCL combined with careful analysis of the data and good planning has made this possible. This should be able to assist with developing rate laws that are useful both for research planning and for developing flight system sizing relationships that can help optimize hybrid rocket concepts for trade studies. The successful approach of this DOE and test setup is applicable to other propellant combinations as well.

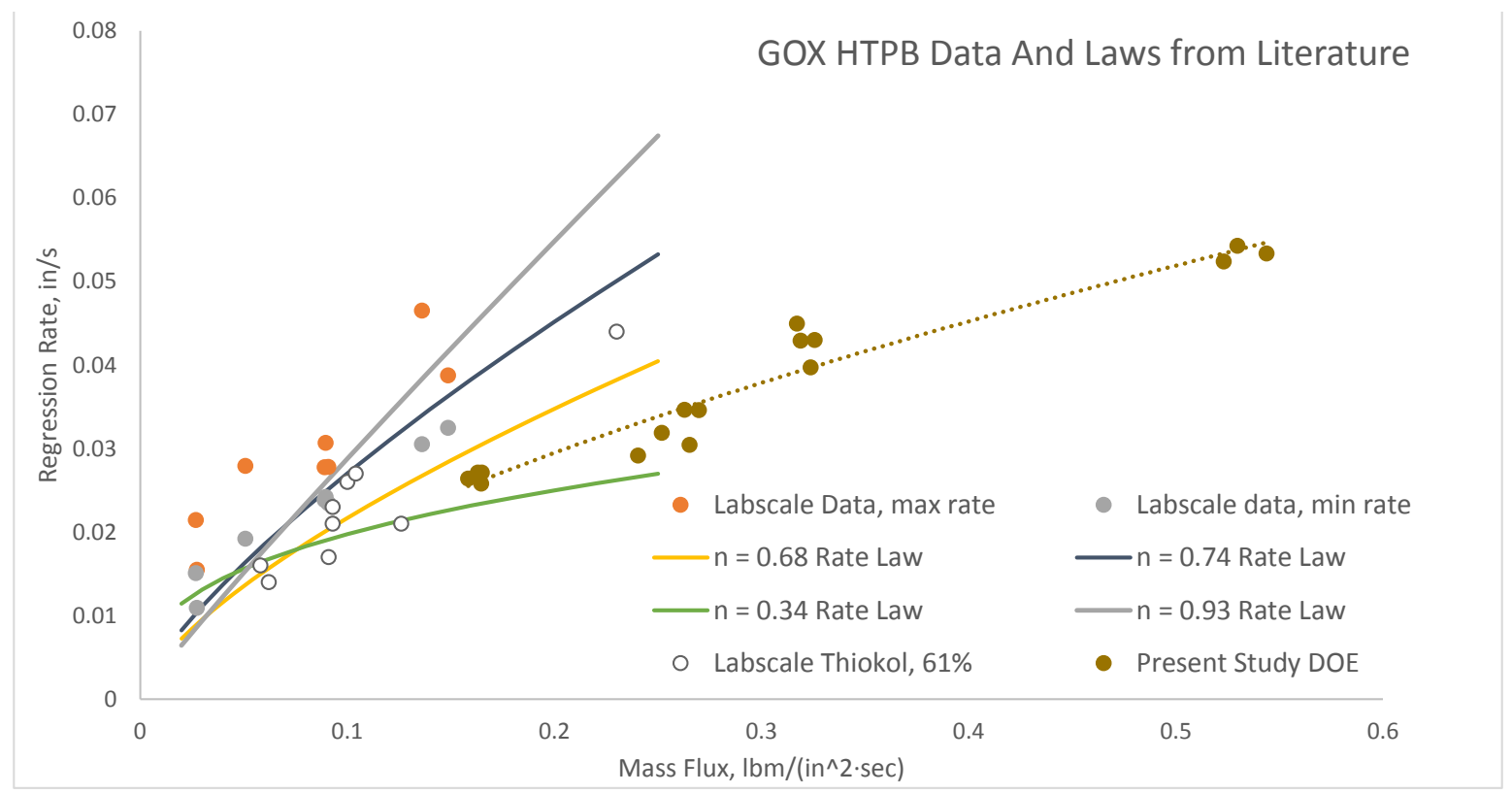


Figure 6. Comparison of data and regression rate correlation from present study with certain literature values.

G. References

1 Sutton, G., and Biblarz, O. (and Boardman, T. for Appendix 4), Rocket Propulsion Elements, $7^{\text {th }}$ Ed., New York: John Wiley and Sons, 2001.

2 Karabeyoglu, M. Arif, Cantwell, B., and Zilliac, G., "Development Of Scalable Space-Time Averaged Regression Rate Expressions For Hybrid Rockets," AIAA-2005-3544, 41st AIAA/ASME/ASEE Joint Propulsion Conference, Tucson AZ, July 2005.

3 Lund, G., and Kliger, J., "Labscale Solid Rocket Combustion Simulator (LSRCS) Final Report,” TWR-40330, Thiokol Corporation, Brigham City, June 1992.

4 Estey, P., Altman, D., and McFarlane, J., "An Evaluation of Scaling Effects for Hybrid Rocket Motors," AIAA-912517, 27th AIAA/SAE/ASME Joint Propulsion Conference, Sacramento CA, June 1991.

5 Labscale Test Report for the Large Subscale Solid Rocket Combustion Simulator Program, Contract NAS8-39874, NASA MSFC, 30 November 1993 